02,05

\title{
Анизотропия намагниченности текстурированного ВТСП Ві-2223 в сильных магнитных полях
}

\author{
(С Д.М. Гохфельд ${ }^{1,2}$, Д.А. Балаев ${ }^{1,2}$ \\ ${ }^{1}$ Институт физики им. Л.В. Киренского ФИЦ КНЦ СО РАН, \\ Красноярск, Россия \\ ${ }^{2}$ Сибирский федеральный университет, \\ Красноярск, Россия \\ E-mail: gokhfeld@iph.krasn.ru
}

Поступила в Редакцию 13 февраля 2020 г.

В окончательной редакции 13 февраля 2020 г.

Принята к публикации 18 фревраля 2020 г.

Исследованы причины малой величины анизотропии намагниченности объёмных текстурированных образцов, состоящих из сильноанизотропных кристаллитов высокотемпературного сверхпроводника $(\mathrm{Bi}, \mathrm{Pb})_{2} \mathrm{Sr}_{2} \mathrm{Ca}_{2} \mathrm{Cu}_{3} \mathrm{O}_{x}$ (Bi-2223). Установлено, что наблюдаемая анизотропия определяется неупорядоченным расположением кристаллитов Вi-2223 в образце. Измеренная величина анизотропии текстурированного образца позволяет определить значение магнитного угла, являющегося характеристикой упорядоченности кристаллитов.

Ключевые слова: критический ток, анизотропия, петля гистерезиса намагниченности, BSCCO.

DOI: 10.21883/FTT.2020.07.49465.031

\section{1. Введение}

Значительная анизотропия физических свойств кристаллов высокотемпературных сверхпроводников (ВТСП) связана с их слоистой кристаллической структурой. В монокристаллах ВТСП анизотропия критического тока в плоскости $a b$ незначительна, в отличие от сильной анизотропии свойств относительно направления оси $c$ и в плоскости $a b$. Анизотропия сверхпроводящего кристалла определяется коэффициентом $\gamma=J_{c, a b} / J_{c, c}$, где $J_{c, a b}$ - это значение плотности критического тока в плоскости $a b$, а $J_{c, c}$ - это значение плотности критического тока вдоль $c$-оси кристалла. ВТСП Ві-2223 известен наиболее сильной анизотропией, значение коэффициента $\gamma$ для него превышает $150[1,2]$.

В поликристаллических ВТСП неупорядоченность анизотропных кристаллитов (здесь и далее под термином „кристаллит“ понимается пластинчатая гранула, микрокристалл) приводит к уменьшению плотности транспортного критического тока. Создание упорядоченной структуры кристаллитов, т.е. текстуры, в поликристаллическом ВТСП позволяет увеличить транспортный критический ток, приблизив его плотность к значениям $J_{c, a b}$ [3]. Однако для текстурированных сверхпроводников Вi-2212 и Вi-2223 значение анизотропии, определяемое из транспортных и магнитных измерений [4-7], составляет всего 2-5, что намного меньше соответствующего значения анизотропии для монокристаллов.

Анизотропия магнитных свойств текстурированного сверхпроводника в первую очередь определяется внутренней кристаллической анизотропией. Определяемая в измерениях величина анизотропии может зависеть от формы образца или гранул и от неупорядоченности кристаллитов.

В настоящей работе мы рассматриваем влияние различных механизмов на анизотропию магнитных свойств и устанавливаем причину слабой анизотропии текстурированных ВТСП на основе висмута. Для этого проводится анализ петель гистерезиса намагниченности текстурированных Вi-2223, представленных ранее в работах $[8,9]$.

\section{2. Магнитная анизотропия текстурированного Ві-2223}

Образцы для измерений намагниченности выпиливались в форме куба с гранями $1.6 \mathrm{~mm}$. Использовались два варианта ориентации внешнего магнитного поля $H$ по отношению к преимущественному направлению кристаллитов в текстурированном образце: магнитное поле направлено перпендикулярно плоскости текстурирования $(H \| c)$ и вдоль плоскости текстурирования $(H \| a b)$. Намагниченность $M$ текстурированного Вi-2223 измерялась в интервале магнитных полей \pm 6 Т в температурах от 4.2 до $80 \mathrm{~K}$ [9]. Для текстурированного Вi-2223 с добавлением серебра [8] измерения проводились в полях $\pm 6 \mathrm{~T}$ в $4.2 \mathrm{~K}$ и $\pm 0.15 \mathrm{~T}$ в $80 \mathrm{~K}$.

На вставках к рис. 1, $a, b$ приведены петли гистерезиса намагниченности Вi-2223 при ориентации внешнего поля перпендикулярно плоскости текстурирования и параллельно плоскости текстурирования. Петли гистерезиса обладают типичными особенностями для кривых намагниченности „нежестких“ сверхпроводников второго рода. Осевая асимметрия (относительно оси $H$ ) увеличивается с ростом температуры. Максимальный 

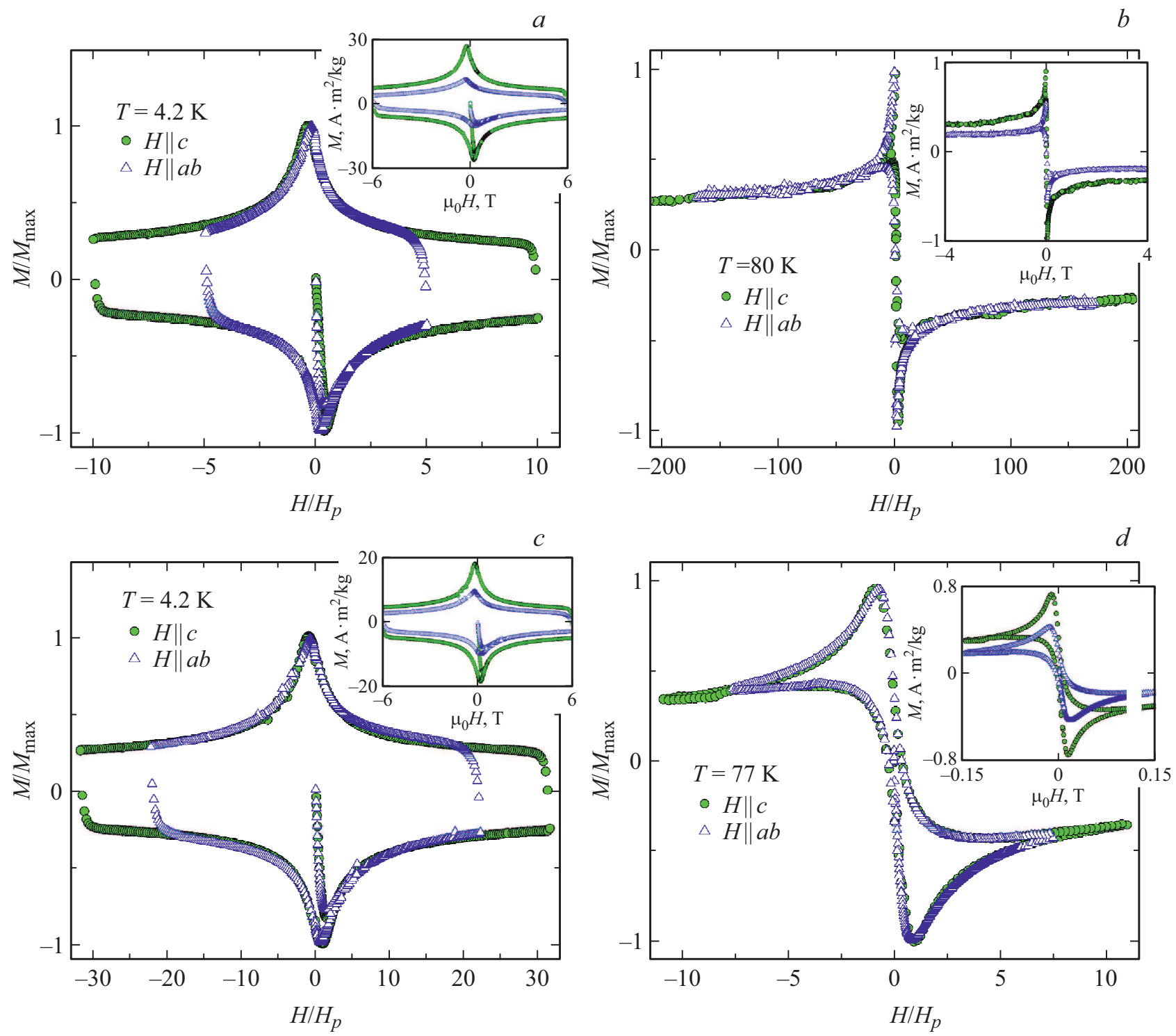

Рис. 1. Магнитный гистерезис Ві-2223 при Н $\| c$ и при $\mathbf{H} \| a b$. Скейлинг петель гистерезиса намагниченности текстурированного $\mathrm{Bi}-2223(a, b)$ Скейлинг петель гистерезиса намагниченности текстурированного Bi-2223 $+\mathrm{Ag}(c, d)$. На вставках показаны петли гистерезиса при разных ориентациях внешнего магнитного поля.

диамагнитный отклик достигается во внешнем магнитном поле, примерно равном полю полного проникновения. Поле полного проникновения определяется как значение внешнего поля, при котором сливаются участок начального намагничивания и полная петля гистерезиса. При увеличении температуры $T$ от 4.2 до $80 \mathrm{~K}$, поле полного проникновения $H_{p, H \| c}$ экспоненциально уменьшается от 0.6 до $0.01 \mathrm{~T}$, а $H_{p, H \| a b}$ изменяется от 1.2 до $0.02 \mathrm{~T}$ (здесь и далее нижний индекс $H \| c$ или $H \| a b$ показывает ориентацию внешнего поля относительно плоскости текстурирования образца). Такое же экспоненциальное уменьшение с температурой было отмечено для плотности критического тока [9]. Аналогично, в этом же температурном интервале происходит уменьшение модуля максимального диамагнитного отклика
$M_{\max , H \| c}$ от $26.5 \mathrm{~A} \cdot \mathrm{m}^{2} / \mathrm{kg}$ до $1.2 \mathrm{~A} \cdot \mathrm{m}^{2} / \mathrm{kg}$ и $M_{\max , H \| a b}$ от $10.9 \mathrm{~A} \cdot \mathrm{m}^{2} / \mathrm{kg}$ до $0.6 \mathrm{~A} \cdot \mathrm{m}^{2} / \mathrm{kg}$.

\section{3. Анализ}

\section{1. Скейлинг кривых намагниченности}

Влияние ориентации анизотропного сверхпроводника во внешнем магнитном поле на термодинамические параметры успешно описывается теорией ГинзбургаЛандау для случая анизотропных сверхпроводников [10]. Параметры анизотропного кристалла, зависящие от ориентации магнитного поля, можно соотнести с параметрами эквивалентного изотропного кристалла, находящегося в эффективном поле $H^{*}$. Эффективное поле $H^{*}$ 
определяется выражением

$$
H^{*}=H\left(\gamma^{-2} \sin ^{2} \theta+\cos ^{2} \theta\right)^{0.5}
$$

где $\theta$ - угол между направлением внешнего поля и c-осью кристалла. Согласно [11], полевые зависимости намагниченности анизотропного образца при $H \| c$ и при $H \| a b$ связаны между собой соотношением

$$
M_{H \| c}(H)=\gamma M_{H \| a b}(\gamma H) .
$$

Скейлинговое соотношение (2) используется для определения коэффициента анизотропии $\gamma$ из магнитных измерений.

На рис. 1, $a, b$ представлены петли гистерезиса намагниченности текстурированного Вi-2223 в $T=4.2 \mathrm{~K}$ и $80 \mathrm{~K}$ в координатах $M / M_{\max }$ от $H / H_{p}$. В этих координатах кривые намагниченности при $H \| c$ и $H \| a b$ совпадают. При увеличении температуры значения $M_{\max }$ и $H_{p}$ уменьшаются. Однако, во всех температурах не изменяется отношение $H_{p, H\|a b / p, H\| c}=2.0 \pm 0.3$. Отношение $M_{\max , H \| c} / M_{\max , H \| a b}$ незначительно уменьшается (от 2.5 до 2.2 при увеличении температуры от 4.2 до $80 \mathrm{~K}$ ). Скейлинговое соотношение (2) удовлетворительно описывает влияние ориентации внешнего магнитного поля на намагниченность при достаточно малом значении $\gamma \approx 2-2.5$. Аналогичное поведение петель гистерезиса намагниченности наблюдается для текстурированного Вi-2223 с добавлением серебра. На рис. 1, $c, d$ показан скейлинг петель гистерезиса $M(H)$ в температуре 4.2 и $77 \mathrm{~K}$. Для этого материала оцененное значение коэффициента анизотропии оказалось еще меньше: $\gamma \approx 1.4-1.7$.

Следует отметить, что сравнимые малые значения анизотропии ранее были получены при исследовании магнитосопротивления этих же материалов [7,12]. Хотя для гранулярных ВТСП магнитосопротивление во многом определяется межгранульными границами [13-16], в случае сильноанизотропных ВТСП, какими являются Bi-2223, диссипация внутри гранул также имеет место в умеренных и сильных магнитных полях $[12,17,18]$. При направлении внешнего поля перпендикулярно плоскости текстурирования $(H \| c)$ сопротивление примерно в 2.4 раза больше, чем при направлении поля параллельно плоскости текстурирования $(H \| a b)$. Таким образом, анизотропия, определяемая из петель гистерезиса намагниченности и из магнитосопротивления текстурированных образцов, оказывается намного меньше анизотропии кристалла Вi-2223.

\section{2. Влияние формы кристаллитов на магнитную анизотропию}

Анизотропия намагниченности также зависит от размеров сверхпроводника в направлениях параллельно и перпендикулярно приложенному магнитному полю. Согласно модели критического состояния [19], намагниченность образца пропорциональна плотности критического тока, циркулирующего в плоскости, перпендикулярной магнитному полю. Для случая анизотропного сверхпроводника модификация модели критического состояния была предложена в работе [20]. При $H \| a b$ высота гистерезиса намагниченности $\Delta M$ анизотропного кристалла определяется следующими формулами

$$
\begin{gathered}
\Delta M_{H \| a b}=J_{c, a b} \frac{d}{2}\left(1-\frac{d}{3 l} \frac{J_{c, a b}}{J_{c, c}}\right) \\
\text { при } \quad J_{c, a b} / J_{c, c}<l / d ; \\
\Delta M_{H \| a b}=J_{c, c} \frac{l}{2}\left(1-\frac{l}{3 d} \frac{J_{c, c}}{J_{c, a b}}\right) \\
\text { при } \quad J_{c, a b} / J_{c, c}>l / d>1,
\end{gathered}
$$

где $d$ это толщина кристалла вдоль оси $c, l$ это минимальный размер кристалла в $a b$ плоскости. При $H \| c$ ток циркулирует в $a b$ плоскости, и высота петли определяется как

$$
\Delta M_{H \| c}=J_{c, a b} l / 3
$$

Проведем оценку анизотропии намагниченности, которая должна соответствовать анизотропии кристалла Вi-2223. Исследуемая текстурированная керамика состоит из упорядоченных плоских кристаллитов с толщиной $\sim 1 \mu \mathrm{m}$ вдоль оси $c$ и поперечными размерами $\sim 5-10 \mu \mathrm{m}$ в $a b$ плоскости [8,9]. Намагниченность поликристаллического образца определяется циркуляцией токов на масштабе гранул [21,22], следовательно, в формулах (3-5) следует подставлять размеры кристаллитов. Принимаем $l \approx 7 \mu \mathrm{m}, d \approx 1 \mu \mathrm{m}, J_{c, a b} / J_{c, c}>150$, так что $J_{c, a b} / J_{c, c}>l / d$. Из формул (4) и (5) выражаем анизотропию намагниченности

$$
\frac{\Delta M_{H \| c}}{\Delta M_{H \| a b}}=\frac{2}{3} \frac{J_{c, a b}}{J_{c, c}}\left(1-\frac{l}{3 d} \frac{J_{c, c}}{J_{c, a b}}\right)^{-1} .
$$

Подставив значения размеров и плотности критического тока кристаллитов, получаем $\Delta M_{H \| c} / \Delta M_{H \| a b}>101$. Однако петли гистерезиса намагниченности Ві-2223 (рис. 1, $a$ ) имеют намного менышее отношение высот гистерезиса: $\Delta M_{H \| c} / \Delta M_{H \| a b} \approx 2.5$. Таким образом, пластинчатая форма кристаллитов не объясняет наблюдаемую анизотропию намагниченности текстурированного образца.

\section{3. Влияние разориентации кристаллитов}

Неидеальная упорядоченность кристаллитов в текстурированном образце эквивалентна отклонению внешнего магнитного поля от выделенных направлений 0 и $90^{\circ}$ в анизотропном сверхпроводящем кристалле. Рассмотрим, как влияет такое отклонение на петлю гистерезиса намагниченности и величину $H_{p}$. На рис. 2 схематично показан образец, состоящий из упорядоченных пластинчатых кристаллитов (слева) и магнитный отклик анизотропного сверхпроводящего кристалла в 


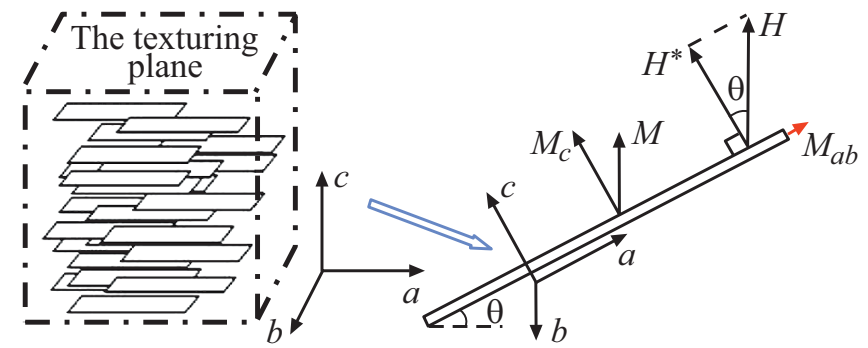

Pис. 2. Схема магнитного отклика анизотропного кристаллита, повернутого на угол $\theta$ относительно плоскости текстурирования. Ориентация осей $a, b, c$ кристаллита отличается от ориентации осей текстурированного образца. Показан случай ориентации внешнего поля $H \| a b$ относительно осей образца.

скошенном поле (справа). $M_{c}$ - это намагниченность, которую создают токи, циркулирующие в $a b$ плоскости. $M_{a b}$ - это намагниченность, которая создается токами, циркулирующими перпендикулярно $a b$ плоскости кристалла. Предполагаем далее $M_{a b} \ll M_{c}$, так что намагниченностью $M_{a b}$ можно пренебречь (случай сильной анизотропии). Действительно, в сильноанизотропных сверхпроводниках токи протекают преимущественно по $a b$ плоскостям кристаллитов [7,23-25]. Соответственно намагниченность сверхпроводника определяется циркуляцией токов в $a b$-плоскостях кристаллитов.

При измерениях определяется проекция намагниченности на ось, соответствующую направлению поля. В то же время, индуцированные в $a b$ плоскости токи зависят от проекции поля $H$ на ось $c$. Для анизотропной пластины, показанной на рис. 2, измеряемые значения намагниченности меньше, чем намагниченность пластины в направлении, параллельной ее оси $c: M_{H \| c}=M_{c} \cos \theta$ и $M_{H \| a b}=M_{c} \sin \theta$. Проекция поля $H$ на ось $c$ определяется как $H_{H \|_{c}^{*}}^{*}=$ $=H \cos \theta$ и $H_{H \| a b}^{*}=H \sin \theta$. Отсюда следует соотношение $M_{H \| c}(H \cos \theta) / \cos \theta=M_{H \| a b}(H \sin \theta) / \sin \theta$, которое можно записать в виде:

$$
M_{H \| c}(H)=k_{\gamma} M_{H \| a b}\left(k_{\gamma} H\right),
$$

где $k_{\gamma}=\operatorname{ctg} \theta$.

Назовем магнитным углом разупорядоченности $\theta^{*}$ усредненное по модулю отклонение кристаллитов от плоскости текстурирования в текстурированном поликристаллическом сверхпроводнике [26]. Скейлинговая формула (5) $\mathrm{c} k \gamma=\operatorname{ctg} \theta^{*}$ описывает соотношение между полевыми зависимостями намагниченности текстурированного образца при $H \| c$ и при $H \| a b$. Отметим, что формула (5) совпадает с скейлинговой формулой (2) при $k_{\gamma}=\gamma$, хотя соответствующие причины анизотропии намагниченности различны. Магнитный угол $\theta^{*}$ характеризует разупорядоченность кристаллитов. Неупорядоченному поликристаллическому образцу соответствует $\theta^{*}=45^{\circ}$. Для текстурированного образца с $\theta^{*}<1^{\circ}$ влияние внутренней анизотропии кристаллитов перестает быть пренебрежимо малым. При $\theta^{*}=0$ возникает расходимость формулы (5), что связано с использованным приближением сильной анизотропии $\gamma=\infty$.

Скейлинг для текстурированного Вi-2223 с $k_{\gamma}=2.5$ (данные на рис. $1, a, b$ ) соответствует магнитному углу $\theta^{*}=21.8^{\circ}$. Такое немалое значение свидетельствует о дисперсии ориентационного распределения кристаллитов в исследуемом материале [27]. Скейлинг для текстурированного Bi-2223 + Ag с $k_{\gamma}=1.7$ (данные на рис. $1, c, d)$ соответствует магнитному углу $\theta^{*}=30.5^{\circ}$. В этом образце кристаллиты менее упорядочены, чем в образце текстурированного Bi-2223 $\left(\theta^{*}=21.8^{\circ}\right)$. Неидеальная упорядоченность кристаллитов является причиной уменьшения диамагнитного сигнала в исследуемых образцах. Необходимо отметить, что фактор Лотгеринга [28], полученный из дифрактограмм исследуемых образцов, равен $0.97 \pm 0.01$ [8]. Такое значение соответствует магнитному углу $\sim 1^{\circ}$ и высокой степени упорядоченности [27]. Различная степень упорядоченности, получаемая из рентгеноструктурных и магнитных данных, может быть вызвана зависимостью ориентационного распределения кристаллитов от расстояния от поверхности образца. По-видимому, высокая упорядоченность кристаллитов реализовалась только на поверхности исследуемых образцов, а во внутреннем объеме ориентация кристаллитов менее упорядочена. Создание сверхпроводящих лент с высокой упорядоченностью кристаллитов Вi-2223 позволяет получить материалы с анизотропией намагниченности более 20 и улучшенными токонесущими свойствами [29,30].

Для исследованных образцов наилучшее совпадение полевых зависимостей в разных температурах достигается при использовании несколько отличающихся значений коэффициентов скейлинга для осей абсцисс и ординат: $M_{\max , H \| c} / M_{\max , H \| a b} \approx 2.5$ и $H_{p, H \| a b} / H_{p, H \| c} \approx 2$ (рис. $1, a, b)$. Различие коэффициентов скейлинга для осей абсцисс и ординат может быть объяснено особой структурой вихрей Абрикосова в слоистом сверхпроводнике. Магнитные вихри в ВТСП являются pancakeвихрями [31]. В скошенном поле сердцевины pancakeвихрей могут сдвигаться в разных слоях ВТСП кристалла, что может приводить к уменьшению угла между внешним полем и вихревыми нитями в образце.

\section{4. Циркуляционный радиус}

Приведенное в разделе 3.3 рассмотрение применимо, если циркуляция токов осуществляется в $a b$ плоскостях кристаллитов. При этом масштаб циркуляции токов должен быть независим от ориентации внешнего магнитного поля. Характерный масштаб циркуляции токов оценивается из расширенной модели критического состояния [21]. Согласно этой модели, несимметричное расположение нижней и верхней ветвей петли гистерезиса связано с отношением глубины поверхностной области с равновесной намагниченностью к радиусу циркуляции экранирующего то- 
ка $R_{c}$. Соответствующее выражение было получено в работе [22]: $\lambda / R_{c} \approx 1-\left|\Delta M\left(H_{p}\right) / 2 M \uparrow\left(H_{p}\right)\right|^{1 / 3}$, здесь $\Delta M=M \downarrow-M \uparrow, M \uparrow$ и $M \downarrow-$ значения намагниченности при увеличении и уменьшении внешнего магнитного поля, $\lambda$ - глубина проникновения магнитного поля. Из асимметрии петель гистерезиса намагниченности, показанных на рис. $1, a$, найдено $\lambda / R_{c} \approx 0.045$ для любой ориентации образца. Одинаковая асимметрия свидетельствует в пользу того, что масштаб циркуляции тока в образце одинаков при $H \| c$ и $H \| a b$. Для значения $\lambda=150 \mathrm{~nm}[3]$ получаем оценку $\approx 5 \mu \mathrm{m}$. Такой масштаб соответствует размеру кристаллитов в $a b$ плоскости. Таким образом, в больших полях циркуляция тока происходит преимущественно в $a b$ плоскостях отдельных кристаллитов как для ориентации $H \| c$, так и $H \| a b$. Соответственно, для любой из рассматриваемых ориентаций, намагниченность текстурированного образца определяется проекцией магнитного отклика от токов, циркулирующих в $a b$-плоскостях неидеально упорядоченных кристаллитов (рис. 2). В работе [32] также было установлено определяющее влияние пиннинга вихрей в $a b$-плоскости кристаллитов на магнитные свойства лент Bi-2223.

\section{4. Заключение}

В результате проведенного анализа было установлено, что независимо от ориентации внешнего магнитного поля, циркуляция токов происходит в $a b$-плоскости кристаллитов Вi-2223. Наблюдаемая анизотропия магнитного гистерезиса текстурированных образцов Bi-2223 несет информацию о разупорядоченности кристаллитов Bi-2223, а не об их внутренней анизотропии. Предложен способ определения эффективной степени текстурирования из измерений магнитного гистерезиса при $H \| c$ и $H \| a b$. Значительное увеличение диамагнитного отклика при $H \| c$ может быть достигнуто оптимизацией упорядоченности кристаллитов в образце.

\section{Конфликт интересов}

Авторы заявляют, что у них нет конфликта интересов.

\section{Список литературы}

[1] J.C. Martínez, S.H. Brongersma, A. Koshelev, B. Ivlev, P.H. Kes, R.P. Griessen, D.G. de Groot, Z. Tarnavski, A.A. Menovsky. Phys. Rev. Lett. 69, 15, 2276 (1992).

[2] O.V. Kharissova, E.M. Kopnin, V.V. Maltsev, N.I. Leonyuk, L.M. Leon-Rossano, I.Yu. Pinus, B.I. Kharisov. Crit. Rev. Solid State 39, 4, 253 (2014).

[3] G. Wang, M.J. Raine, D.P. Hampshire. Supercond. Sci. Technol. 30, 10, 104001 (2017).

[4] A.D. Caplin, L.F. Cohen, M.N. Cuthbert, M. Dhalle, D. Lacey, G.K. Perkins, J.V. Thomas. IEEE Trans. Appl. Supercond. 5, 2, 1864 (1995).

[5] G.C. Han. Phys. Rev. B 52, 2, 1309 (1995).
[6] G.C. Han, C.K. Ong. Phys. Rev. B 56, 17, 11299 (1997).

[7] Д.М. Гохфельд, Д.А. Балаев, С.В. Семенов, М.И. Петров. ФTT 57, 11, 2090 (2015).

[8] M.I. Petrov, I.L. Belozerova, K.A. Shaikhutdinov, D.A. Balaev, A.A. Dubrovskii, S.I. Popkov, A.D. Vasil'ev, O.N. Mart'yanov. Supercond. Sci. Technol. 21, 10, 105019 (2008).

[9] D.M. Gokhfeld, D.A. Balaev, M.I. Petrov, S.I. Popkov, K.A. Shaykhutdinov, V.V. Val'kov. J. Appl. Phys. 109, 3, 033904 (2011).

[10] J.R. Clem. Supercond. Sci. Technol. 11, 10, 909 (1998).

[11] Z. Hao, J.R. Clem. Phys. Rev. B 46, 9, 5853 (1992).

[12] D.A. Balaev, S.I. Popkov, S.V. Semenov, A.A. Bykov, K.A. Shaykhutdinov, D.M. Gokhfeld, M.I. Petrov. Physica C 470, 1, 61 (2010).

[13] D.A. Balaev, S.I. Popkov, E.I. Sabitova, S.V. Semenov, K.A. Shaykhutdinov, A.V. Shabanov, M.I. Petrov. J. Appl. Phys. 110, 9, 093918 (2011).

[14] С.В. Семёнов, Д.А. Балаев, М.А. Почекутов, Д.А. Великанов. ФТТ 59, 7, 1267 (2017).

[15] D.A. Balaev, S.V. Semenov, M.A. Pochekutov. J. Appl. Phys. 122, 12, 123902 (2017).

[16] В.В. Деревянко, Т.В. Сухарева, В.А. Финкель. ФТТ 60, 3, 465 (2018).

[17] Д.А. Балаев, А.А. Быков, С.В. Семенов, С.И. Попков, А.А. Дубровский, К.А. Шайхутдинов, М.И. Петров. ФТТ 53, 865 (2011).

[18] D.A. Balaev, S.I. Popkov, S.V. Semenov, A.A. Bykov, E.I. Sabitova, A.A. Dubrovskiy, K.A. Shaikhutdinov, M.I. Petrov. J. Supercond. Nov. Magn. 24, 2129 (2011).

[19] C.P. Bean. Phys. Rev. Lett. 8, 6, 250 (1962).

[20] E.M. Gyorgy, R.B. van Dover, K.A. Jackson, L.F. Schneemeyer, J.V. Waszczak. Appl. Phys. Lett. 55, 3, 283 (1989).

[21] Д.М. Гохфельд. ФТТ 56, 12, 2298 (2014).

[22] Д.М. Гохфельд. Письма в ЖТФ 45, 2, 3 (2019).

[23] J.H. Cho, M.P. Maley, J.O. Willis, J.Y. Coulter, L.N. Bulaevskii, P. Haldar, L.R. Motowidlo. Appl. Phys. Lett. 64, 22, 3030 (1994).

[24] B. Hensel, G. Grasso, R. Flükiger. Phys. Rev. B 51, 21, 15456 (1995).

[25] A. Diaz, J. Maza, F. Vidal. Phys. Rev. B 55, 2, 1209 (1997).

[26] B. Lehndorff, M. Hortig, H. Piel. Supercond. Sci. Technol. 11, 11, 1261 (1998).

[27] R. Furushima, S. Tanaka, Z. Kato, K. Uematsu. J. Ceram. Soc. Jpn. 118, 10, 921 (2010).

[28] F.K. Lotgering. J. Inorg. Nucl. Chem. 9, 2, 113 (1959).

[29] A. Lascialfari, S. De Gennaro, A. Peruzzi, C. Sangregorio. J. Phys. D 31, 17, 2098 (1998).

[30] G. Grasso, M.R. Cimberle, P. Guasconi, I. Pallecchi, C. Ferdeghini. Supercond. Sci. Technol. 12, 12, 1108 (1999).

[31] R.G. Mints, V.G. Kogan, J.R. Clem. Phys. Rev. B 61, 2, 1623 (2000).

[32] M. Kiuchi, E.S. Otabe, T. Matsushita, T. Kato, T. Hikata, K. Sato. Physica C 260, 3-4, 177 (1996).

Редактор К.В. Емцев 